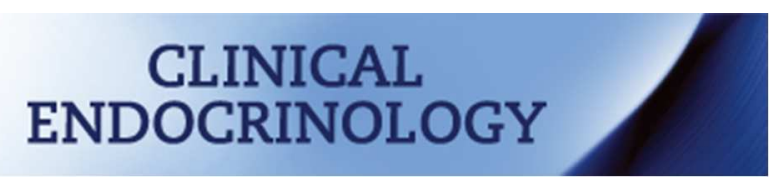

Serum fibroblast growth factor 21 as a biomarker is superior to other adipokines in predicting incident diabetes

\begin{tabular}{|r|l|}
\hline Journal: & Clinical Endocrinology \\
\hline Manuscript ID & CEN-2016-000696.R1 \\
\hline Manuscript Type: & 3 Original Article - Australia, Japan, SE Asia \\
\hline Date Submitted by the Author: & n/a \\
\hline Complete List of Authors: & $\begin{array}{l}\text { Woo, YC; University of Hong Kong, Department of Medicine } \\
\text { LEE, CHI HO; Queen Mary Hospital, Hong Kong, Medicine } \\
\text { Fong, CHY; Queen Mary Hospital, Department of Medicine } \\
\text { Xu, Aimin; University of Hong Kong, Department of Medicine } \\
\text { Tso, Annette WK; University of Hong Kong, Department of Medicine } \\
\text { Cheung, Bernard MY; University of Hong Kong, Department of Medicine } \\
\text { Lam, Karen; University of Hong Kong, Department of Medicine }\end{array}$ \\
\hline Key Words: & $\begin{array}{l}\text { Adipokines < Hormones/related: < Adipose tissue, Diabetes mellitus < } \\
\text { Conditions: < Pancreas, Fibroblast growth factor 21 }\end{array}$ \\
\hline
\end{tabular}




\title{
Serum fibroblast growth factor 21 as a biomarker is superior to other adipokines in predicting incident diabetes
}

Yu Cho Woo ${ }^{1}$, Chi Ho Lee ${ }^{1,2}$, Carol HY Fong ${ }^{1}$, Aimin Xu ${ }^{1,2,3}$, Annette WK Tso ${ }^{1}$, Bernard MY Cheung ${ }^{1,2,3}$, Karen SL Lam ${ }^{1,2,3}$

Department of Medicine ${ }^{1}$, State Key Laboratory of Pharmaceutical Biotechnology ${ }^{2}$ and Research Centre of Heart, Brain, Hormone and Healthy Aging ${ }^{3}$, The University of Hong Kong, Hong Kong

\author{
Abbreviated Title: FGF21 as a biomarker for diabetes prediction \\ Key words: FGF21, adipokines, diabetes \\ Word Counts: 2785 \\ Corresponding author: Karen Lam (ksllam@hku.hk) or Bernard Cheung (mycheung@hku.hk) \\ Address: Department of Medicine, University of Hong Kong, Queen Mary Hospital, 102 Pokfulam \\ Road, Hong Kong, China \\ Telephone: +852 2255-4783; Fax: +852 2816-2863 \\ Funding: Research Grant Council, Hong Kong (CRF HKU02/12R) \\ Disclosure Statement: The authors have nothing to disclose
}




\section{Summary \\ Objective:}

Fibroblast growth factor 21 (FGF21) improves glucose and lipid metabolism but high circulating levels are found in type 2 diabetes, suggesting FGF21 resistance. Serum FGF21 predicts incident diabetes but its performance, compared to established and emerging predictors, is not known. We aimed to study the performance of FGF21 in diabetes prediction, relative to other adipokines and established risk factors including 2-hour plasma glucose $(2 \mathrm{hG})$ during the oral glucose tolerance test (OGTT).

\section{Design/Participants/Measurements:}

We studied 1380 non-diabetic subjects from the Hong Kong Cardiovascular Risk Factor Prevalence Study using the second visit (2000-2004) as baseline when serum levels of FGF21 and other adipokines were measured. Glycaemic status was assessed by OGTT. Incident diabetes was defined as fasting glucose level $(\mathrm{FG}) \geq 7 \mathrm{mmol} / \mathrm{L}$ or $2 \mathrm{hG} \geq 11.1 \mathrm{mmol} / \mathrm{L}$ or use of anti-diabetic agents, at subsequent visits.

\section{Results:}

123 participants developed diabetes over 9.0 years (median). On multivariable logistic regression analysis, FGF21 ( $\mathrm{p}=0.003)$, adipocyte-fatty acid binding protein $(\mathrm{p}=0.003)$ and adiponectin $(\mathrm{p}=0.035)$ were independent predictors of incident diabetes. FGF21 had the best change in log likelihood when added to a diabetes prediction model (DP) based on age, family history, smoking, hypertension, BMI, dyslipidemia and FG. It also improved the area under ROC curve (AUROC) of DP from 0.797 to $0.819(\mathrm{P}=0.0072)$, rendering its performance comparable to the " $\mathrm{DP}+2 \mathrm{hG}$ " model (AUROC $=0.838$, $\mathrm{P}=0.19)$.

\section{Conclusions:}

As a biomarker for diabetes prediction, serum FGF21 appeared to be superior to other adipokines and, on its own, could be considered as an alternative to the OGTT.

Word count: 249 


\section{Introduction}

Fibroblast growth factor 21 (FGF21) is secreted predominantly by the liver but also by other tissues involved in glucose and lipid metabolism, such as the adipose tissue, pancreas and skeletal muscle ${ }^{1}$, with the contribution from the adipose tissue being much increased in obesity. Multiple beneficial effects of FGF21 on insulin sensitivity, glucose and lipid homeostasis have been observed in animal models ${ }^{2}$. FGF21 is possibly also bioactive in humans as treatment of obese diabetic subjects with FGF21-based therapies resulted in beneficial effects on body weight, plasma lipoprotein profile and serum adiponectin levels ${ }^{3,4}$, and reduced fasting insulin ${ }^{3}$. Circulating FGF21 levels, however, were elevated in obesity ${ }^{5}$, as well as in obesity-related diseases such as dyslipidemia, type 2 diabetes (T2DM), non-alcoholic fatty liver disease, carotid atherosclerosis and coronary artery disease ${ }^{6-10}$. Raised serum FGF21 levels in these diseases may represent a compensatory response to the underlying metabolic stresses ${ }^{1}$, including insulin resistance/hyperinsulinemia, or may be secondary toFGF21 resistance. Indeed, impaired FGF21 signalling in adipocytes has been demonstrated in mice with diet-induced obesity ${ }^{11}$. The above clinical observations have suggested the potential role of FGF21 as a biomarker for obesity-related diseases. Notably, high serum FGF21 levels have been shown to predict the development of T2DM in two 5-year prospective studies ${ }^{12}$.

The dysregulated secretion, and hence altered circulating levels of various adipokines in obesity ${ }^{13}$, has raised the possibility that serum levels of such adipokines can be used as biomarkers of obesityrelated diseases such as T2DM. In the ARIC study of 4 US communities, it was found that the measurement of adiponectin and leptin, but not interleukin- 6 and retinol acid binding protein 4 , could provide a modest improvement on the prediction of T2DM by conventional risk factors ${ }^{14}$. In the Chinese population, we previously found that adiponectin, tumour necrosis factor-alpha (TNF- $\alpha$ ) or its soluble receptor tumour necrosis factor-alpha receptor 2 (TNF- $\alpha$ R2), and adipocyte fatty acid binding protein (A-FABP), could all independently predict incident diabetes ${ }^{15,16}$. We also found that the combined use of serum adiponectin and TNF- $\alpha$ R2 levels, when added to conventional risk factors, had comparable performance to 2-hour plasma glucose $(2 \mathrm{hG})$ during the oral glucose tolerance test (OGTT), in predicting incident diabetes ${ }^{15}$. With the increasing information on FGF21 as a metabolic regulator in humans ${ }^{3,4}$ and its role as a novel biomarker for predicting T2DM ${ }^{8,12}$, it appeared timely that we should assess its performance in the prediction of incident diabetes, in comparison to other adipokines and established predictors of T2DM, including the OGTT. This was conducted using the 9-year prospective data from the Hong Kong Cardiovascular Risk Factor Prevalence Study (CRISPS).

\section{Materials and Methods:}

Subjects were recruited from CRISPS, a long-term, population-based, prospective study on the development of cardiovascular risk factors in Hong Kong. In 1995-1996 (CRISPS1), 2,895 unrelated Chinese subjects were invited randomly by their telephone numbers to undergo a detailed assessment 
17. Subjects returned for reassessment visits in 2000-2004 (CRISPS2), 2005-2008 (CRISPS3) and 2010-2012 (CRISPS4). Details of medical history taking, anthropometric and biochemical parameters measurements were described elsewhere ${ }^{18}$. Subjects attended the visits in the morning and all blood taking was performed at 9am after an overnight fast to avoid the effect of diurnal changes and meals on circulating level of the adipokines ${ }^{19}$. Serum levels of the adipokines potentially predictive of T2DM were measured from stored serum samples collected at CRISPS2 hence this visit was used as the baseline of the current study. Subjects who had been started on anti-diabetic medications, or with fasting glucose $(\mathrm{FG}) \geq 7 \mathrm{mmol} / \mathrm{L}$ or $2-\mathrm{hG} \geq 11.1 \mathrm{mmol} / \mathrm{L}$ were considered to have diabetes. Subjects without diabetes at baseline visit were followed for their glycemic status at CRISPS3 and CRISPS4. The incident diabetes group included all subjects with incident diabetes when screened at CRISPS3 or CRISPS4, whilst subjects in the non-diabetes group were those who remained non-diabetic at CRISPS4. Hypertension was defined as systolic blood pressure $\geq 140 \mathrm{mmHg}$, diastolic blood pressure $\geq 90 \mathrm{mmHg}$, or on hypertensive treatment. The presence of dyslipidaemia was defined as: triglycerides $\geq 1.7 \mathrm{mmol} / \mathrm{L}, \mathrm{HDL}$ cholesterol $<1.0 \mathrm{mmol} / \mathrm{L}$ for men and 1.3 for women; LDL cholesterol $\geq 3.4 \mathrm{mmol} / \mathrm{L}$, or on lipid-lowering treatment. Insulin resistance was estimated using the homeostasis model assessment index of insulin resistance (HOMA-IR), calculated by the formula (FG in $\mathrm{mmol} / \mathrm{L} \mathrm{x}$ fasting insulin in $\mathrm{mIU} / \mathrm{L} / 22.5$ ). The study was approved by the ethics committee of the Faculty of Medicine, University of Hong Kong. Informed consent was obtained from all subjects.

Detailed information on the measurement of serum A-FABP, TNF- $\alpha$ R2 and total adiponectin levels were described previously ${ }^{15}$. TNF- $\alpha-\mathrm{R} 2$ was measured as a surrogate marker for TNF- $\alpha$ because soluble TNF- $\alpha-\mathrm{R} 2$ can be measured in frozen plasma with greater sensitivity and reliability than TNF- $\alpha{ }^{20}$. Serum leptin was measured using a commercial ELISA kit (BioVendor Laboratory Medicine, Brno, Czech). Serum FGF21 was measured with an in-house ELISA kit (Antibody and Immunoassay Services, University of Hong Kong) as previously described ${ }^{5,21}$. The intra- and interassay variations were $4.5 \%$ and $6.8 \%$ respectively.

All statistical analyses were performed with SPSS Statistics 19 (SPSS, Chicago, IL). Results were presented as mean $\pm \mathrm{SD}$ or median with interquartile range (IQR) as appropriate. For data that were not normally distributed, natural logarithmic transformation was applied before analyses. In univariate analyses, variables were compared between groups by one-way ANOVA for continuous data and Chisquare test for categorical data respectively. Biomarkers including A-FABP, adiponectin, leptin and TNF- $\alpha$ R2 with circulating levels showing gender-specific dimorphisms were sex-adjusted. They were categorised as high or low level with reference to their optimal cut-offs identified by Youden index ${ }^{22}$. Multivariable logistic regression was used to test for significant independent variables for incident diabetes. To compare the relative performance of biomarkers, a basic model based on conventional risk factors and common biochemical parameters, which were independent predictors or of clinical relevance, was constructed. Log-likelihood ratio tests were used to compare the change in log 
likelihood (-2LL) before and after addition of biomarkers to the basic model using multivariable logistic regression analysis. The biomarker with the best -2LL was tested for the change in the area under the curve of receiver operating characteristics (AUROC) when added to the basic model and compared with the AUROC with $2 \mathrm{hG}$ added to the basic model. Differences in AUROC were assessed using Delong's test ${ }^{23}$.

\section{Results}

123 subjects developed incident diabetes over a median of 9.0 years (IRQ: 6.6 - 9.7). Those who developed diabetes were older, with higher body mass index (BMI), waist circumference (WC), FG, fasting insulin and HOMA-IR (all $\mathrm{p}<0.001$ ) at baseline visit. More of them were had a history of smoking, family history of diabetes (both $\mathrm{p}=0.005$ ), central obesity, hypertension and dyslipidaemia (all $\mathrm{p}<0.001$ ). The levels of all biomarkers were significantly different between the incident diabetes and non-diabetes group in univariate analysis (Table 1). The optimal cut-offs of the biomarkers were as follows: $178.2 \mathrm{pg} / \mathrm{mL}$ for FGF21; $29.8 \mathrm{ng} / \mathrm{mL}$ in men and $22.4 \mathrm{ng} / \mathrm{mL}$ in women for A-FABP; 6.19 $\mu \mathrm{g} / \mathrm{mL}$ in men and $6.03 \mu \mathrm{g} / \mathrm{mL}$ in women for adiponectin; $2051.1 \mathrm{ng} / \mathrm{ml}$ in men and $2134.6 \mathrm{ng} / \mathrm{ml}$ in women for TNF- $\alpha$ R2; $4.666 \mathrm{ng} / \mathrm{mL}$ for men and $10.227 \mathrm{ng} / \mathrm{mL}$ for women for leptin. On multiple logistic regression analysis, smoking history, family history of diabetes, WC and BMI were the clinical parameters independently predictive of diabetes, whilst FG was the independent biochemical predictor. Of the biomarkers, high FGF21 and A-FABP, but not TNF- $\alpha$ R2 and leptin, were independent predictors of diabetes, whereas high adiponectin was associated with a reduced risk of incident diabetes (Table 2). Addition of FGF21 to the basic diabetes prediction model (DP) consisting of age, smoking history, BMI, family history, hypertension, dyslipidaemia and FG resulted in the best $-2 \mathrm{LL}$ of the model, followed by A-FABP and adiponectin, whilst addition of TNF- $\alpha$ R2 or leptin did not result in significant changes in -2LL (Table 3). The AUROC of using FGF21 alone in diabetes prediction was 0.708 . When added to DP, the model consisting of established diabetes risk factors, FGF21 significantly improved the AUROC of DP from 0.797 to 0.819 ( $\mathrm{p}=0.0072$ ). Likewise, $2 \mathrm{hG}$ significantly improved the AUROC of DP to 0.838 . However, there was no significant difference observed in the AUROC of the two models: "FGF21+DP" versus " $2 \mathrm{hG}+\mathrm{DP}$ " (0.819 vs. 0.838 , $\mathrm{p}=0.19$ ) (Table 4, Figure 1). Using FGF21 levels in the form of continuous data instead of applying the cut-off value did not affect the results (Table $3 \& 4$ ). Addition of adiponectin or A-FABP to the same DP model did not show significant improvement in the AUROC (Table 4). In our previous report, the combination of ADP + TNF- $\alpha$ R2 had a significant improvement when added to the basic model ${ }^{15}$. However, in this cohort with a longer follow up duration and more cases of incident diabetes, adding the same combination of biomarkers to the DP did not show significant improvement 
in AUROC (0.807 vs. 0.797, $\mathrm{P}=0.09$ ), in keeping with the superior predictive performance of serum FGF21 as a biomarker of incident diabetes, even when used on its own.

\section{Discussions}

It has been well proven that development of diabetes can be effectively delayed or prevented in a costeffective manner in high risk subjects with impaired glucose tolerance ${ }^{24}$. Despite requiring the inconvenient and cumbersome OGTT, $2 \mathrm{hG}$ remains a very strong predictor for diabetes development, which predicts diabetes on its own and provides additional value to other prediction models ${ }^{15,25}$. In this paper, we showed that FGF21, a novel metabolic regulator of glucose and lipid metabolism and a potential therapeutic agent for metabolic disorders, could also be a useful biomarker for the prediction of incident diabetes. As a single biomarker, it enhanced significantly the prediction of diabetes development by established risk factors, with a performance that was comparable to $2 \mathrm{hG}$, and appeared to be superior to other adipokines previously studied in our CRISPS cohort.

Obesity indices are good predictors of incident diabetes. Simple diabetes prediction models usually rely on obesity indices as their prerequisite parameters. In our cohort, the obesity indices, BMI and WC, were also independent predictors. It is however noteworthy that only $20 \%$ or fewer obese white people will progress to diabetes over subsequent years ${ }^{26}$. Likewise, in this long term prospective Chinese cohort, only $12.1 \%$ subjects with $\mathrm{BMI} \geq 23$ and $20.5 \%$ of subjects with BMI $\geq 27.5$ developed diabetes in 9 years' time. Hence prediction models usually also include other parameters to improve the overall performance. In the basic model we constructed for testing the performances of different biomarkers, we did not include both BMI and WC because of their strong correlation. BMI was preferred to WC in the model because the measurement of body weight and height should be more precise than WC, which is heavily influenced by the anatomic location of measurement ${ }^{27}$. Taking our basic model as an example, incorporation of other clinical risk factors, like age, hypertension, family history, smoking history as well as readily measurable metabolic parameters, including fasting glucose and lipid profiles to BMI, already resulted in a reasonably good predictive model with an AUROC at 0.797 . Hence it has been argued that a prediction model based on simple and clinically available variables might do even better than performing the OGTT ${ }^{25}$. In our cohort, however, the addition of the $2 \mathrm{hG}$ measurement to the multivariable model, which included FG and dyslipidaemia in addition to clinically available risk factors, still further increased the AUC of the prediction model, in this and a previous study ${ }^{15}$. On the other hand, being an inconvenient test lacking in reproducibility, the OGTT is far from ideal as a screening tool and a replacement with comparable predictability of diabetes should be most welcome. We did demonstrate in our previous study with a shorter follow up period and fewer incident diabetes cases, that the addition of a combination of two obesity-related biomarkers, namely adiponectin and TNF- $\alpha \mathrm{R} 2$, could be comparable to $2 \mathrm{hG}$ in 
improving diabetes prediction by established risk factors, dyslipidaemia and $\mathrm{FG}^{15}$. These observations may suggest that $2 \mathrm{hG}$ remains a strong predictor for future diabetes because it represents the part of pathophysiology of T2DM that cannot be reflected by FG, but can be attributed at least partially to the effects of circulating adipokines. The findings in the current study suggest that FGF21, as a single obesity-related biomarker, appears to have a prediction performance that is closest to $2 \mathrm{hG}$, compared to others that have been studied in our population.

FGF21 has multiple beneficial effects on insulin sensitivity, glucose and lipid homeostasis in animal models. However, raised FGF 21 levels have been generally observed in obesity and conditions associated with obesity or increased insulin resistance. While insulin resistance is well known to appear before the onset of diabetes, FGF21 resistance also precedes dysglycaemia $(7,12)$. The raised FGF21 levels in these conditions may be due to a compensatory response to metabolic stresses resulted from increased circulating free fatty acids and insulin, which are known to stimulate increased FGF21 expression ${ }^{1}$. Moreover, the adipose tissue inflammation in obesity subjects which involves the c-Jun NH2-terminal kinase 1 pathway could result in the suppression of $\beta$-Klotho expression by TNF- $\alpha$ and hence impaired FGF21 action in adipocytes ${ }^{11}$. FGF21 is also the upstream regulator of adiponectin. It induces expression and secretion of adiponectin in adipocytes to confer the glucose-lowering and insulin-sensitizing effects ${ }^{28}$. Hence FGF21 resistance in obesity can potentially lead to insulin resistance through hypoadiponectinaemia. Whereas the role of hypoadiponectinaemia in diabetes development is well established ${ }^{13-15}$, the mechanism linking A-FABP with glucose homeostasis is less clear. A recent study, however, has demonstrated that A-FABP is a secreted adipokine regulating hepatic glucose production, and its immune-neutralization in vivo led to reduced gluconeogenesis and the correction of diabetic phenotype in obese mice ${ }^{29}$. Our study suggested that leptin and TNF- $\alpha$, both pro-inflammatory adipokines with increased expression in obesity ${ }^{13}$, are not by themselves the major players in diabetes development in our cohort. In a nutshell, FGF21 has been found to be involved in the interplay with various other hormones in the pathogenesis of T2DM, rendering it a good candidate as a biomarker for diabetes prediction and a possible alternative to the inconvenient OGTT. FGF21 was also found to be useful as a biomarker in other diseases related to obesity. Serum FGF21 levels were found to be significantly increased in non-alcoholic fatty liver disease and were positively correlated with intrahepatic triglyceride. Measurement of FGF21 might have the additional benefit of detecting mild steatosis, while the diagnostic sensitivity of ultrasonography decreased sharply if the degree of steatosis was less than $30 \%$ on biopsy ${ }^{30}$. There may be additional advantage of measurement of FGF21 in assessing or predicting other diseases related to obesity and insulin resistance.

Our study has the strengths of being a long-term study with prospective data on incident diabetes over 9 years in a genetically homogenous Chinese population. Our findings should be of considerable value in diabetes prediction in the populous major cities of Mainland China. The glycaemic status of 
all participants, except those who had been taking anti-diabetic agents, was reliably verified by OGTT. We did not use HbAlc as a diagnostic criterion for diabetes as the diagnostic criteria have changed over time with the HbA1c criterion only being accepted after 2011. Had the HbA1c criterion been adopted, an additional number of subjects would have been screened to have diabetes as shown by our results at CRISPS $4{ }^{31}$. On the other hand, the data of this paper is also limited by the drawback of a very long-term prospective study as we unavoidably had participants lost to follow up due to various reasons. Participants who returned for follow-up visits up till CRISPS4 might well be the more health conscious subjects and might not be truly representative of the general population. Application of the FGF21 level for diabetic risk prediction requires integration with other risk factors to formulate a risk score which requires multi-steps calculations and may be inconvenient to use when compared with prediction models based on simple measurable parameters. However, this clumsy calculation procedure is not difficult to overcome due to the relatively easy accessibility to computers or even smartphones nowadays. Lastly, our participants were middle-aged individuals with a mean age of 50.3 and the youngest participant was 30 years old at CRISPS2 baseline. Our conclusions may not be applicable to younger individuals.

In summary, we have demonstrated that, as a single biomarker, FGF21 adds value to established risk factors when used in the construction of diabetes prediction models. In this regard, its performance appears to be superior to other obesity-related biomarkers and is comparable to the 2-hour post OGTT glucose. Further studies in other populations are warranted to validate its use as a biomarker to identify high risk subjects for interventional measures to prevent diabetes development. 


\section{References}

1 Woo, Y.C., Xu, A., Wang, Y. \& Lam, K.S. (2013) Fibroblast growth factor 21 as an emerging metabolic regulator: clinical perspectives. Clin Endocrinol (Oxf) 78, 489-496.

2 Kharitonenkov, A. \& DiMarchi, R. (2015) FGF21 Revolutions: Recent Advances Illuminating FGF21 Biology and Medicinal Properties. Trends Endocrinol Metab 26, 608-617.

3 Gaich, G., Chien, J.Y., Fu, H., Glass, L.C., Deeg, M.A., Holland, W.L., Kharitonenkov, A., Bumol, T., Schilske, H.K. \& Moller, D.E. (2013) The effects of LY2405319, an FGF21 analog, in obese human subjects with type 2 diabetes. Cell Metab 18, 333-340.

4 Talukdar, S., Zhou, Y., Li, D., Rossulek, M., Dong, J., Somayaji, V., Weng, Y., Clark, R., Lanba, A., Owen, B.M., Brenner, M.B., Trimmer, J.K., Gropp, K.E., Chabot, J.R., Erion, D.M., Rolph, T.P., Goodwin, B. \& Calle, R.A. (2016) A Long-Acting FGF21 Molecule, PF-05231023, Decreases Body Weight and Improves Lipid Profile in Non-human Primates and Type 2 Diabetic Subjects. Cell Metab 23, 427-440.

5 Zhang, X., Yeung, D.C., Karpisek, M., Stejskal, D., Zhou, Z.G., Liu, F., Wong, R.L., Chow, W.S., Tso, A.W., Lam, K.S. \& Xu, A. (2008) Serum FGF21 levels are increased in obesity and are independently associated with the metabolic syndrome in humans. Diabetes 57, 1246-1253.

6 Inagaki, T. (2015) Research Perspectives on the Regulation and Physiological Functions of FGF21 and its Association with NAFLD. Front Endocrinol (Lausanne) 6, 147.

7 Li, H., Bao, Y., Xu, A., Pan, X., Lu, J., Wu, H., Lu, H., Xiang, K. \& Jia, W. (2009) Serum fibroblast growth factor 21 is associated with adverse lipid profiles and gamma-glutamyltransferase but not insulin sensitivity in Chinese subjects. J Clin Endocrinol Metab 94, 2151-2156.

8 Chen, C., Cheung, B.M., Tso, A.W., Wang, Y., Law, L.S., Ong, K.L., Wat, N.M., Xu, A. \& Lam, K.S. (2011) High plasma level of fibroblast growth factor 21 is an Independent predictor of type 2 diabetes: a 5.4-year population-based prospective study in Chinese subjects. Diabetes Care 34, 21132115.

9 Chow, W.S., Xu, A., Woo, Y.C., Tso, A.W., Cheung, S.C., Fong, C.H., Tse, H.F., Chau, M.T., Cheung, B.M. \& Lam, K.S. (2013) Serum fibroblast growth factor-21 levels are associated with carotid atherosclerosis independent of established cardiovascular risk factors. Arterioscler Thromb Vasc Biol 33, 2454-2459.

10 Lin, Z., Wu, Z., Yin, X., Liu, Y., Yan, X., Lin, S., Xiao, J., Wang, X., Feng, W. \& Li, X. (2010) Serum levels of FGF-21 are increased in coronary heart disease patients and are independently associated with adverse lipid profile. PLoS One 5, e15534.

11 Diaz-Delfin, J., Hondares, E., Iglesias, R., Giralt, M., Caelles, C. \& Villarroya, F. (2012) TNFalpha represses beta-Klotho expression and impairs FGF21 action in adipose cells: involvement of JNK1 in the FGF21 pathway. Endocrinology 153, 4238-4245.

12 Bobbert, T., Schwarz, F., Fischer-Rosinsky, A., Pfeiffer, A.F., Mohlig, M., Mai, K. \& Spranger, J. (2013) Fibroblast growth factor 21 predicts the metabolic syndrome and type 2 diabetes in Caucasians. Diabetes Care 36, 145-149.

13 Tso, A.W., Xu, A., Chow, W.S. \& Lam, K.S. (2008) Adipose tissue and the metabolic syndrome: focusing on adiponectin and several novel adipokines. Biomark Med 2, 239-252.

14 Raynor, L.A., Pankow, J.S., Duncan, B.B., Schmidt, M.I., Hoogeveen, R.C., Pereira, M.A., Young, J.H. \& Ballantyne, C.M. (2013) Novel risk factors and the prediction of type 2 diabetes in the Atherosclerosis Risk in Communities (ARIC) study. Diabetes Care 36, 70-76.

15 Woo, Y.C., Tso, A.W., Xu, A., Law, L.S., Fong, C.H., Lam, T.H., Lo, S.V., Wat, N.M., Cheung, B.M. \& Lam, K.S. (2012) Combined use of serum adiponectin and tumor necrosis factor-alpha receptor 2 levels was comparable to 2-hour post-load glucose in diabetes prediction. PLoS One 7, e36868.

16 Tso, A.W., Xu, A., Sham, P.C., Wat, N.M., Wang, Y., Fong, C.H., Cheung, B.M., Janus, E.D. \& Lam, K.S. (2007) Serum adipocyte fatty acid binding protein as a new biomarker predicting the 
development of type 2 diabetes: a 10-year prospective study in a Chinese cohort. Diabetes Care 30, 2667-2672.

17 Janus, E.D., Watt, N.M., Lam, K.S., Cockram, C.S., Siu, S.T., Liu, L.J. \& Lam, T.H. (2000) The prevalence of diabetes, association with cardiovascular risk factors and implications of diagnostic criteria (ADA 1997 and WHO 1998) in a 1996 community-based population study in Hong Kong Chinese. Hong Kong Cardiovascular Risk Factor Steering Committee. American Diabetes Association. Diabet Med 17, 741-745.

18 Cheung, B.M., Wat, N.M., Man, Y.B., Tam, S., Thomas, G.N., Leung, G.M., Cheng, C.H., Woo, J., Janus, E.D., Lau, C.P., Lam, T.H. \& Lam, K.S. (2007) Development of diabetes in Chinese with the metabolic syndrome: a 6-year prospective study. Diabetes Care 30, 1430-1436.

19 Yu, H., Xia, F., Lam, K.S., Wang, Y., Bao, Y., Zhang, J., Gu, Y., Zhou, P., Lu, J., Jia, W. \& Xu, A. (2011) Circadian rhythm of circulating fibroblast growth factor 21 is related to diurnal changes in fatty acids in humans. Clin Chem 57, 691-700.

20 Liu, S., Tinker, L., Song, Y., Rifai, N., Bonds, D.E., Cook, N.R., Heiss, G., Howard, B.V., Hotamisligil, G.S., Hu, F.B., Kuller, L.H. \& Manson, J.E. (2007) A prospective study of inflammatory cytokines and diabetes mellitus in a multiethnic cohort of postmenopausal women. Arch Intern Med 167, 1676-1685.

21 Lee, C.H., Hui, E.Y., Woo, Y.C., Yeung, C.Y., Chow, W.S., Yuen, M.M., Fong, C.H., Xu, A. \& Lam, K.S. (2015) Circulating fibroblast growth factor 21 levels predict progressive kidney disease in subjects with type 2 diabetes and normoalbuminuria. J Clin Endocrinol Metab 100, 1368-1375.

22 Perkins, N.J. \& Schisterman, E.F. (2006) The inconsistency of "optimal" cutpoints obtained using two criteria based on the receiver operating characteristic curve. Am J Epidemiol 163, 670-675. 23 DeLong, E.R., DeLong, D.M. \& Clarke-Pearson, D.L. (1988) Comparing the areas under two or more correlated receiver operating characteristic curves: a nonparametric approach. Biometrics 44, 837-845.

24 Ratner, R.E. \& Diabetes Prevention Program, R. (2006) An update on the Diabetes Prevention Program. Endocr Pract 12 Suppl 1, 20-24.

25 Stern, M.P., Williams, K. \& Haffner, S.M. (2002) Identification of persons at high risk for type 2 diabetes mellitus: do we need the oral glucose tolerance test? Ann Intern Med 136, 575-581.

26 Meigs, J.B., Wilson, P.W., Fox, C.S., Vasan, R.S., Nathan, D.M., Sullivan, L.M. \& D'Agostino, R.B. (2006) Body mass index, metabolic syndrome, and risk of type 2 diabetes or cardiovascular disease. J Clin Endocrinol Metab 91, 2906-2912.

27 Mason, C. \& Katzmarzyk, P.T. (2009) Variability in waist circumference measurements according to anatomic measurement site. Obesity (Silver Spring) 17, 1789-1795.

28 Lin, Z., Tian, H., Lam, K.S., Lin, S., Hoo, R.C., Konishi, M., Itoh, N., Wang, Y., Bornstein, S.R., Xu, A. \& Li, X. (2013) Adiponectin mediates the metabolic effects of FGF21 on glucose homeostasis and insulin sensitivity in mice. Cell Metab 17, 779-789.

29 Cao, H., Sekiya, M., Ertunc, M.E., Burak, M.F., Mayers, J.R., White, A., Inouye, K., Rickey, L.M., Ercal, B.C., Furuhashi, M., Tuncman, G. \& Hotamisligil, G.S. (2013) Adipocyte lipid chaperone AP2 is a secreted adipokine regulating hepatic glucose production. Cell Metab 17, 768-778.

30 Li, H., Fang, Q., Gao, F., Fan, J., Zhou, J., Wang, X., Zhang, H., Pan, X., Bao, Y., Xiang, K., Xu, A. \& Jia, W. (2010) Fibroblast growth factor 21 levels are increased in nonalcoholic fatty liver disease patients and are correlated with hepatic triglyceride. J Hepatol 53, 934-940.

31 Woo, Y.C., Cheung, B.M., Yeung, C.Y., Lee, C.H., Hui, E.Y., Fong, C.H., Tso, A.W., Tam, S. \& Lam, K.S. (2015) Cardiometabolic risk profile of participants with prediabetes diagnosed by HbA1C criteria in an urban Hong Kong Chinese population over 40 years of age. Diabet Med. 32,1207-11. 


\section{Figure legends}

Figure 1 Comparisons of AUROCs of different diabetes prediction models.

A: Diabetes Prediction Model (DP) based on Age, body mass index, family history of diabetes, ever smoking history, hypertension, fasting glucose and dyslipidaemia; B: DP + 2h-Glucose; C: DP + FGF21 (with cut-off); D: FGF21 (continuous data); FGF21, fibroblast growth factor 21

12
13
14
15
16
17
18
19
20
21
22
23
24
25
26
27
28
29
30
31
32
33
34
35
36
37
38
39
40
41
42
43
44
45
46
47
48
49
50
51
52
53
54
55
56
57
58
59
60

45

46

47

48

49

50

52

53

54

55

56

57

59

60 
Table 1 Baseline clinical and biochemical characteristics of subjects with and without incident Type 2 diabetes in 9.0 (6.6-9.7) years

\begin{tabular}{|c|c|c|c|}
\hline Baseline variables & Cumulative DM & Non-DM & $\mathrm{p}$-value \\
\hline $\mathrm{N}$ & 123 & 1257 & -- \\
\hline Age, years & $55.2 \pm 10.4$ & $49.8 \pm 10.9$ & $<0.001$ \\
\hline Men, $\%$ & 51.6 & 44.7 & 0.139 \\
\hline Ever smoker, \% & 34.7 & 23.4 & 0.005 \\
\hline Physical activity, \% & 30.8 & 27.4 & 0.430 \\
\hline Family history of diabetes, $\%$ & 25.8 & 16.0 & 0.005 \\
\hline Central Obesity, \% & 47.6 & 23.4 & $<0.001$ \\
\hline Waist circumference, $\mathrm{cm}$ & & & $<0.001 *$ \\
\hline Male & $88.5 \pm 8.89$ & $82.8 \pm 8.31$ & \\
\hline Female & $80.8 \pm 9.05$ & $74.7 \pm 8.57$ & \\
\hline Body Mass Index, $\mathrm{kg} / \mathrm{m}^{2}$ & $25.9 \pm 3.69$ & $23.6 \pm 3.22$ & $<0.001$ \\
\hline Hypertension, \% & 33.9 & 19.3 & $<0.001$ \\
\hline Systolic blood pressure, $\mathrm{mmHg}$ & $128 \pm 19$ & $119 \pm 17$ & $<0.001$ \\
\hline $\begin{array}{l}\text { Diastolic blood pressure, } \\
\mathrm{mmHg}\end{array}$ & $79 \pm 9$ & $74 \pm 10$ & $<0.001$ \\
\hline IGT/IFG, \% & 66.9 & 22.9 & $<0.001$ \\
\hline Fasting glucose, $\mathrm{mmol} / \mathrm{L}$ & $5.45 \pm 0.58$ & $5.01 \pm 0.47$ & $<0.001$ \\
\hline Fasting insulin ${ }^{\dagger}, \mathrm{mIU} / \mathrm{L}$ & $8.9(6.6-15.0)$ & $6.9(5.0-9.7)$ & $<0.001$ \\
\hline HOMA-IR ${ }^{\dagger}$ & $2.30(1.49-2.30)$ & $1.52(1.11-2.19)$ & $<0.001$ \\
\hline Dyslipidemia, $\%$ & 82.3 & 58.9 & $<0.001$ \\
\hline Total cholesterol, $\mathrm{mmol} / \mathrm{L}$ & $5.51 \pm 1.00$ & $5.24 \pm 0.87$ & 0.002 \\
\hline Triglycerides ${ }^{\dagger}, \mathrm{mmol} / \mathrm{L}$ & $1.3(1.0-2.0)$ & $1.1(0.7-1.5)$ & $<0.001$ \\
\hline HDL cholesterol, $\mathrm{mmol} / \mathrm{L}$ & $1.29 \pm 0.33$ & $1.44 \pm 0.38$ & $<0.001$ \\
\hline LDL cholesterol, mmol/L & $3.50 \pm 0.91$ & $3.23 \pm 0.77$ & $<0.001$ \\
\hline Adiponectin $^{\dagger}, \mathrm{ug} / \mathrm{ml}$ & & & $<0.001^{*}$ \\
\hline Men & $4.53(3.04-6.06)$ & $5.90(3.83-9.35)$ & \\
\hline Women & $7.12(4.42-11.3)$ & $8.16(5.76-11.8)$ & \\
\hline $\mathrm{FGF} 21^{\dagger}, \mathrm{pg} / \mathrm{ml}$ & & & $<0.001^{*}$ \\
\hline Men & $234.7(154.1-408.9)$ & $155.3(84.4-261.3)$ & \\
\hline Women & $266.6(186.5-322.6)$ & $131.1(78.1-233.1)$ & \\
\hline 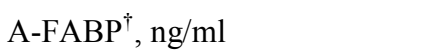 & & & $<0.001 *$ \\
\hline
\end{tabular}




\begin{tabular}{lccc}
\hline Baseline variables & Cumulative DM & Non-DM & p-value \\
\hline Men & $21.8(14.8-31.9)$ & $18.2(13.2-24.4)$ & \\
Women & $29.3(23.4-37.0)$ & $22.0(16.4-30.3)$ & \\
TNF-alpha R2 ${ }^{\dagger}, \mathrm{ng} / \mathrm{ml}$ & & & $<0.001^{*}$ \\
Men & $2153.8(1864.4-2654.6)$ & $1934.9(1683.7-2282.0)$ & \\
$\quad$ Women & $1897.3(1557.6-2335.2)$ & $1758.8(1520.1-2067.4)$ & \\
Leptin ${ }^{\dagger}, \mathrm{ng} / \mathrm{ml}$ & & & $<0.001^{*}$ \\
Men & $5.78(3.21-8.73)$ & $4.01(2.02-6.48)$ & \\
Women & $14.4(10.4-20.7)$ & $11.0(7.75-15.7)$ & \\
& & & \\
\hline
\end{tabular}

Data was presented as mean \pm standard deviation, median (interquartile-range), or percentage as appropriate. *Sex-adjusted p-value; ${ }^{;} \log$ transformed before analysis. Central obesity was defined as waist circumference $\geq$ $90 \mathrm{~cm}$ for men and 80 for women; Hypertension was defined as systolic blood pressure $\geq 140 \mathrm{mmHg}$, diastolic blood pressure $\geq 90 \mathrm{mmHg}$, or on hypertensive treatment. Dyslipidaemia: triglycerides $\geq 1.7 \mathrm{mmol} / \mathrm{L}$, HDL cholesterol $<1.0 \mathrm{mmol} / \mathrm{L}$ for men and 1.3 for women; $\mathrm{LDL}$ cholesterol $\geq 3.4 \mathrm{mmol} / \mathrm{L}$, or on lipid-lowering treatment. 
Table 2 Multivariable logistic regression for significant independent variables predicted the development of DM

\begin{tabular}{lcc}
\hline Baseline variables & Adjusted OR (95\% CI) & p-value \\
\hline Age (years) & $1.02(1.00-1.04)$ & 0.039 \\
Ever smoking & $1.81(1.14-2.85)$ & $\mathbf{0 . 0 1 1}$ \\
Family history of diabetes & $2.37(1.45-3.85)$ & $\mathbf{0 . 0 0 1}$ \\
BMI $\left(\mathrm{kg} / \mathrm{m}^{2}\right)$ & $1.09(1.02-1.16)$ & $\mathbf{0 . 0 0 8}$ \\
HT $(\%)$ & $1.10(0.70-1.72)$ & 0.682 \\
FG $(\mathrm{mmol} / \mathrm{L})$ & $3.63(2.39-5.50)$ & $<\mathbf{0 . 0 0 1}$ \\
Dyslipidemia & $1.61(0.96-2.71)$ & 0.071 \\
FGF21 (pg/ml) & $1.60(1.18-2.16)$ & $\mathbf{0 . 0 0 3}$ \\
A-FABP* (ng/ml) & $2.03(1.28-3.20)$ & $\mathbf{0 . 0 0 3}$ \\
Adiponectin* (ug/ml) & $0.62(0.40-0.97)$ & $\mathbf{0 . 0 3 5}$
\end{tabular}

*Sex specific; BMI, body mass index; HT, hypertension; FG, fasting glucose; FGF21, fibroblast growth factor 21; A-FABP, adipocyte-fatty acid-binding protein. 
1

2

3

4

5

6

7

8

9

10

11

12

13

14

15

16

17

18

19

20

21

22

23

24

25

26

27

28

29

30

31

32

33

34

35

36

37

38

39

40

41

42

43

44

45

46

47

48

49

50

51

52

53

54

55

56

57

58

59

60

Table 3 Log-likelihood ratio tests comparing the change before and after addition of adipokines by using multivariable logistic regression analysis

\begin{tabular}{|c|c|c|c|c|}
\hline Diabetes & Biomarkers & $-2 \mathrm{LL}$ & Change in & p-value \\
\hline Prediction & & & $-2 L L$ from & \\
\hline Model & & & basic model & \\
\hline \multirow{8}{*}{$\begin{array}{l}\text { Age, FH of DM, } \\
\text { smoking, BMI, HT, } \\
\text { FG and dyslipid }\end{array}$} & & 682.562 & -- & \\
\hline & & & & \\
\hline & + FGF21 & & 15.070 & $<0.001$ \\
\hline & $+\mathrm{FGF} 21 *$ & & 21.805 & $<0.001$ \\
\hline & + A-FABP* & & 11.969 & $<0.001$ \\
\hline & + Adiponectin* & & 4.323 & 0.038 \\
\hline & + TNF- $\alpha$ R2* & & 1.558 & 0.212 \\
\hline & + Leptin* & & 1.515 & 0.218 \\
\hline
\end{tabular}

FGF21 was log-transformed before analysis, unless otherwise specified; *Sex-specific optimal cut-off points applied.

-2LL, -2 log Likelihood; FH of DM, family history of diabetes; smoking, history of ever smoking; BMI, body mass index; HT, hypertension; FG, fasting glucose; dyslipid, dyslipidaemia; FGF21, fibroblast growth; A-FABP, adipocyte-fatty acid-binding protein; TNF- $\alpha$ R2: tumour necrosis factoralpha receptor 2 . 
Table 4 Comparisons of AUROCs of different diabetes prediction models.

\begin{tabular}{llccc}
\hline Model & Additional variable(s) & AUROC (95\% CI) & $\begin{array}{c}\text { Delong p-value } \\
{[\text { Referent: }} \\
\text { Base model }]\end{array}$ & $\begin{array}{c}\text { Delong p-value } \\
\text { [Referent: } \\
\text { 2h-Glucose }]\end{array}$ \\
\hline $\begin{array}{l}\text { Age, BMI, Fhx of } \\
\text { DM, Ever smoking, }\end{array}$ & & $0.797(0.773-0.817)$ & Referent & -- \\
HT, FG and Dyslip & & & & \\
\hline & & & & \\
\hline & + FG-Glucose & $0.838(0.817-0.857)$ & $\mathbf{0 . 0 0 1 0}$ & Referent \\
& + FGF21* & $0.813(0.792-0.833)$ & $\mathbf{0 . 0 0 8 3}$ & 0.0622 \\
& + A-FABP* & $0.819(0.798-0.839)$ & $\mathbf{0 . 0 0 7 2}$ & 0.1882 \\
& + ADP* & $0.808(0.786-0.828)$ & 0.0743 & $\mathbf{0 . 0 2 9 0}$ \\
& + TNF- $\alpha R 2^{*}$ & $0.799(0.777-0.820)$ & 0.4319 & $\mathbf{0 . 0 0 2 3}$ \\
& $+A D P^{*}+T N F-\alpha R 2^{*}$ & $0.807(0.785-0.828)$ & 0.0906 & $\mathbf{0 . 0 0 9 9}$ \\
& & $0.804(0.782-0.825)$ & 0.0782 & $\mathbf{0 . 0 2 0 3}$ \\
\hline
\end{tabular}

FGF21 was log-transformed before analysis, unless otherwise specified; *Sex-specific optimal cut-off points applied.

AUROC, Area under the curve of receiver operating characteristics; BMI, body mass index; HT, hypertension; Fhx of DM, family history of diabetes; FG, fasting glucose; dyslip, dyslipidemia; FGF21, fibroblast growth factor 21; A-FABP, adipocyte-fatty acid-binding protein; ADP, adiponectin; TNF- $\alpha$ R2: tumour necrosis factor-alpha receptor 2. 


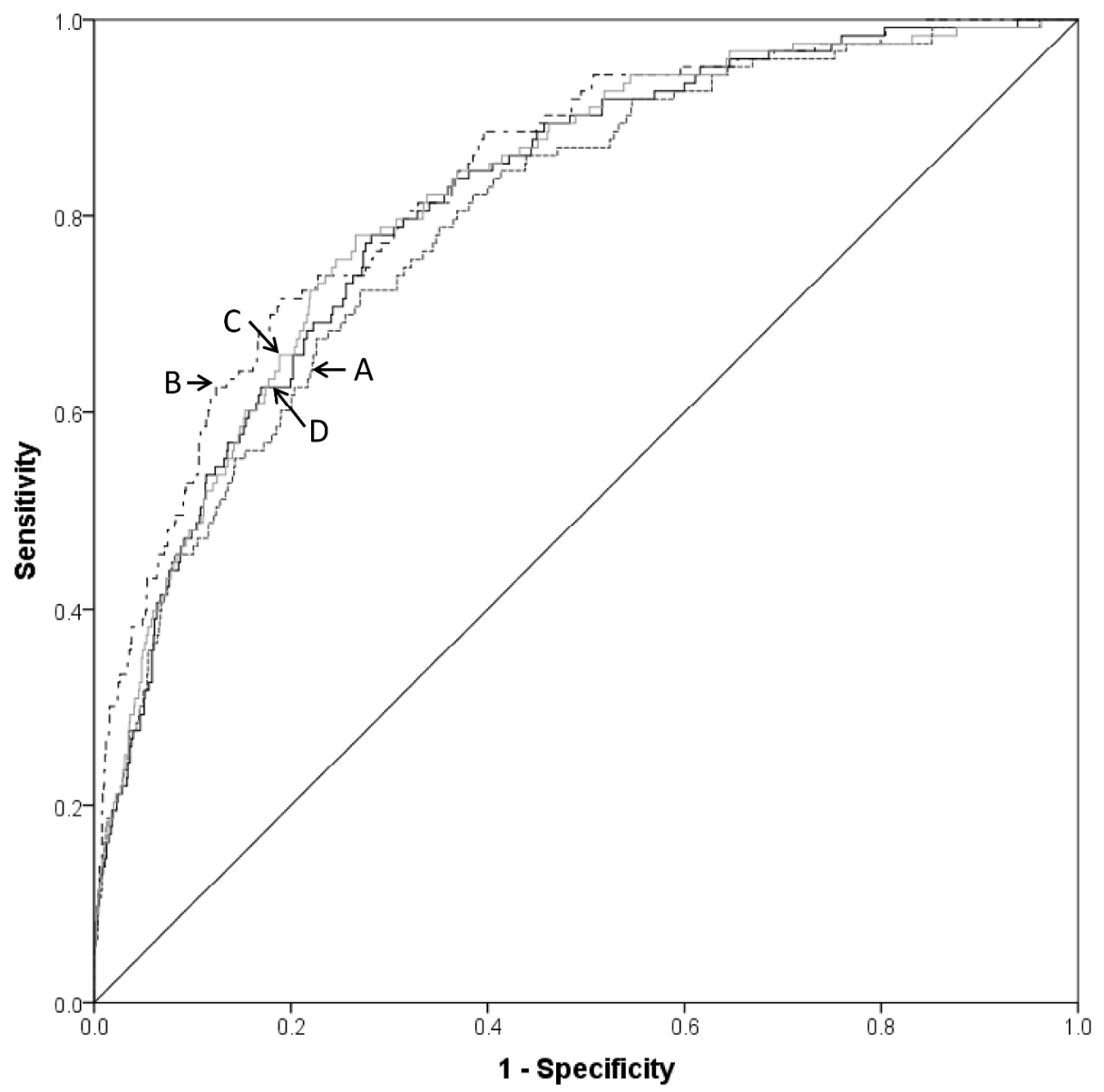

Figure 1 Comparisons of AUROCs of different diabetes prediction models.

A: Diabetes Prediction Model (DP) based on Age, body mass index, family history of diabetes, ever smoking history, hypertension, fasting glucose and dyslipidaemia; B: DP + 2h-Glucose; C: DP + FGF21 (with cutoff); D: FGF21 (continuous data); FGF21, fibroblast growth factor 21 